\title{
Realistische Therapieziele anstreben
}

\author{
Die schmerzhafte diabetische Polyneuropathie ist eine der häu- \\ figsten Spätfolgen des Diabetes mellitus. Sie geht mit einem \\ enormen Leidensdruck einher. Realistische Ziele der Therapie \\ sind neben der Schmerzreduktion eine Verbesserung der allge- \\ meinen Aktivität und des Schlafs.
}

14\% aller Diabetiker entwickeln eine schmerzhafte Polyneuropathie (PN). „Doch der Schmerz ist nicht allein das Problem, dazu kommen häufig Angst und Depression", sagte Dr. Christoph Maier vom Uniklinikum Bergmannsheil in Bochum. Viele betroffene Diabetiker erhielten keine adäquate Schmerztherapie. Bei der Gabe eines Analgetikums müssten gerade bei Diabetikern auch die Nebenwirkungen berücksichtigt werden, vor allem bei Niereninsuffizienz. Dies gelte besonders für nicht-steroidale Antirheumatika, die bei Diabetikern mit Polyneuropathie viel zu oft eingesetzt würden. Aber auch Paracetamol ist nach neueren Erkenntnissen für Diabetiker mit kardiovaskulären oder renalen Problemen bedenklich.

Bevor man eine medikamentöse Schmerztherapie einsetzt, sollte man sich darüber im Klaren sein, welche realistischen Ziele erreicht werden können. „Absolute Schmerzfreiheit werden Sie bei kaum einem Patienten erreichen", so Maier. Doch eine Schmerzreduktion um 30-50\%, eine Besserung des Schlafs und der Lebensqualität, die Erhaltung der sozialen Aktivität und der Arbeitsfähigkeit sollten angestrebt werden.

Für die symptomatische Behandlung der schmerzhaften PN wird in den Leitlinien neben den Antikonvulsiva Gabapentin bzw. Pregabalin und Opioiden als Mittel der ersten Wahl auch der selektive Serotonin-Noradrenalin-Wiederaufnahmehemmer Duloxetin (Cymbalta) empfohlen. Die Analgesie der Substanz beruhe auf einer Modulation der schmerzhemmenden noradrenergen und serotonergen Signale in den absteigenden Rückenmarksbahnen, so Maier.

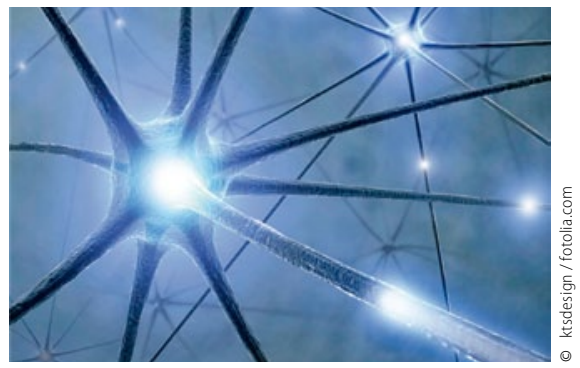

Schmerzimpulse in Neuronen lassen sich bei Polyneuropathie meist nicht ganz bekämpfen.

Die schnelle und starke Wirksamkeit von Duloxetin wurde in mehrere kontrollierten Studien bei schmerzhafter DPN belegt, wobei bereits innerhalb der ersten Woche eine Wirkung belegt wurde. „Die analgetische Wirkung von Duloxetin ist unabhängig von seiner antidepressiven", so Maier. Es würden auch Alltagsaktivitäten und Lebensfreude gesteigert. Die analgetische Wirkung sei vergleichbar mit der von Pregabalin. Vorteilhaft im Vergleich zu Opioiden sei, dass keine Sedierung oder Verstärkung schlafbezogener Atmungsstörungen auftrete.

Dr. med. Peter Stiefelhagen, Hachenburg

Quelle: Satellitensymposium bei der DDG-Jahrestagung am 16.5.2012 in Stuttgart, Veranstalter: Lilly.

\section{DPP-4-Hemmer auch hier anwendbar}

\section{Typ-2-Diabetiker sind meist älter und niereninsuffizient, was Hypoglykämien fördert und den Metformin-Einsatz limitiert. DPP-4-Hemmer bieten hier eine sinnvolle Therapieoption.}

Auch über 65-jährigen Diabetiker, die bereits andere Antidiabetika anwenden, profitieren vom DPP-4-Hemmer Linagliptin (Trajenta ${ }^{\circ}$, wie eine gepoolte Analyse von sieben kontrollierte Studien mit insgesamt 1331 Patienten belegt [1]. Die Patienten erhielten zur laufenden antidiabetischen Therapie - etwa 60\% der Teilnehmer bekamen schon zuvor zwei oder mehr antidiabetische Wirkstoffe - Linagliptin, das in Deutschland nicht verfügbar ist. Es brachte eine zusätzliche $\mathrm{HbA}_{1 c^{-}}$ Senkung um durchschnittlich $0,63 \%$, ohne dass vermehrt unerwünschte Wirkungen aufgetreten wären. Insbesondere die Hypoglykämie-Rate war im Trend sogar niedriger als mit Placebo.

Eine zweite gepoolte Analyse von zwei Studien hatte ähnliche Ergebnisse für mit Insulin oder Insulinanaloga vorbehandelte ältere Diabetiker ergeben, nämlich eine $\mathrm{HbA}_{1 \mathrm{c}}$-Senkung um $0,77 \%$ bei placeboähnlichem Nebenwirkungs- und Hypoglykämie-Risiko [2].

Linagliptin ist derzeit der einzige DPP4-Inhibitor, der auch Typ-2-Diabetikern mit Nierenfunktionsstörungen ohne Dosisanpassung verordnet werden kann, daran erinnerte Prof. Per-Henrik Groop von der Universität Helsinki. Diese Patienten sind nicht nur kardiovaskulär besonders gefährdet, sondern haben auch ein hohes Risiko für schwere Hypoglykämien.

Groop präsentierte eine gepoolte Analyse kontrollierter Phase-3-Studien mit Linagliptin. Sie bezog 277 Diabetiker mit milder Nephropathie ein, die alle einen ACE-Hemmer oder $\mathrm{AT}_{1}$-Blocker nahmen. In der Analyse konnte nach 24 Wochen Therapie ein um 29\% stärkerer Rückgang der Albumin-Ausscheidung als mit Placebo gezeigt werden $(\mathrm{p}=0,03)$. Der Rückgang war zudem größer als die $\mathrm{HbA}_{1 c}$-Senkung es hätte erwarten lassen, so Groop. Derzeit läuft eine Studie, die diese Ergebnisse prospektiv bestätigen soll.

Manuela Arand, Berlin

1. Patel et al. EASD 2012, Poster 850

2. Woerle et al. EASD 2012, Poster 848.

Quelle: Pressekonferenz bei der EASD-Jahrestagung am 1. 10.2012 in Berlin, Veranstalter: Boehringer Ingelheim und Lilly, 